\title{
Heat conduction in rotating relativistic stars
}

\author{
S. K. Lander ${ }^{1 \star}$ and N. Andersson ${ }^{2}$ \\ ${ }^{1}$ Nicolaus Copernicus Astronomical Centre, Polish Academy of Sciences, Bartycka 18, PL-00-716 Warsaw, Poland \\ ${ }^{2}$ Mathematical Sciences and STAG Research Centre, University of Southampton, Southampton SO17 1BJ, UK
}

Accepted 2018 June 26. Received 2018 June 20; in original form 2018 March 30

\begin{abstract}
In the standard form of the relativistic heat equation used in astrophysics, information propagates instantaneously, rather than being limited by the speed of light as demanded by relativity. We show how this equation none the less follows from a more general, causal theory of heat propagation in which the entropy plays the role of a fluid. In deriving this result, however, we see that it is necessary to make some assumptions which are not universally valid: the dynamical time-scales of the process must be long compared with the explicitly causal physics of the theory, the heat flow must be sufficiently steady, and the space-time static. Generalizing the heat equation (e.g. restoring causality) would thus entail retaining some of the terms we neglected. As a first extension, we derive the heat equation for the space-time associated with a slowly-rotating star or black hole, showing that it only differs from the static result by an additional advection term due to the rotation, and as a consequence demonstrate that a hotspot on a neutron star will be seen to be modulated at the rotation frequency by a distant observer.
\end{abstract}

Key words: accretion, accretion discs-conduction-gravitation-stars:- neutron-stars: rotation.

\section{INTRODUCTION}

Treating heat propagation in general relativity (GR) is a necessary ingredient for the quantitative modelling of compact objects. It becomes important during the collapse of a massive star into either a neutron star or black hole (Misner \& Sharp 1964; Govender, Maharaj \& Maartens 1998; Woosley, Heger \& Weaver 2002; Sekiguchi 2010). It is needed to understand neutron-star cooling from birth, through the rapid proto-neutron-star phase (Burrows \& Lattimer 1986) to the slower, secular evolution of mature neutron stars (Van Riper 1991; Aguilera, Pons \& Miralles 2008). It is also important in modelling short-time-scale cooling following outbursts from accreting neutron stars (Cumming et al. 2017), for accretion physics around black holes (Yuan \& Narayan 2014; Ressler et al. 2015), and in the very late stages of a binary neutron-star inspiral (Shibata, Taniguchi \& Uryu 2005).

The standard relativistic heat equation is acausal, predicting that information propagates instantaneously, and thus violating a basic tenet of relativity that nothing can travel faster than light. It is now well established that causality can be restored in a natural way, by treating entropy as a fluid whose dynamics couple to those of the medium (e.g. the various fluid species of a neutron star) (LopezMonsalvo \& Andersson 2011). Relativistic thermal dynamics is naturally expressed in this multifluid framework, and can be applied in problems on short time-scales where the finite-speed propagation

^E-mail: skl@camk.edu.pl of entropy is important, or in situations where the space-time is highly dynamic - like the merger of compact objects. In its full form it is, however, likely to be too computationally complex for many practical purposes.

Here we show how the usual form of the relativistic heat equation, for a spherical space-time, may be recovered from the multifluid framework in a natural way. Along the way we show that it is necessary to make a few assumptions - probably safe ones for secular processes in a neutron-star or black hole environment, but not necessarily in every astrophysical situation. This therefore gives a diagnostic of when the familiar relativistic heat equation is not applicable: if any of these terms is not negligible. Having established the non-rotating result, we generalize our approach to find the heat equation governing a slowly-rotating star, keeping the terms of linear order in the rotation rate (which include frame dragging). We drop second-order rotational terms (which cause the space-time to deviate from sphericity), but note that these have been explored in the context of neutron-star cooling by Miralles, Van Riper \& Lattimer (1993) and Negreiros, Schramm \& Weber (2017).

This paper is aimed at an astrophysics audience, and so is intended to assume no specialist knowledge of the reader. For this reason, we begin with a brief review of the theory of relativistic thermal dynamics and the foliation of space-time for numerical simulations, in order to make our discussion self-contained. We then derive, in turn, Fourier's law and the heat equation; in each case beginning with the non-rotating result and then exploring the effect of slow rotation. We conclude with a discussion of the rotational modulation of a neutron-star hotspot seen by a distant observer. 


\section{RELATIVISTIC THERMAL DYNAMICS}

\subsection{The problem of causality in heat conduction}

The heat equation is so familiar that it is easy to forget one conceptually unsatisfactory feature of it. Although this paper is concerned with heat conduction in GR, let us begin in Newtonian gravity. The heat equation states that a temperature distribution $T$ evolves as

$\frac{\partial T}{\partial t}=-\frac{1}{C_{V}} \nabla \cdot \boldsymbol{Q}$,

where $C_{V}$ is the volumetric heat capacity. The heat flux $\boldsymbol{Q}$ is, in turn, given by Fourier's law:

$Q=-\kappa \nabla T$,

where $\kappa$ is the thermal conductivity. Substituting (2) into (1), however, we see that the resulting heat equation is parabolic - and so the characteristic propagation speed is infinite. Even in Newtonian physics, it is unreasonable to expect some physical quantity to be transmitted instantaneously, and efforts to rectify this deficiency in the heat equation go back decades. A natural reference point is the Cattaneo equation, in which causality is introduced - albeit in a phenomenological manner - through a time-dependent term in Fourier's law:

$\mathfrak{t} \frac{\partial \boldsymbol{Q}}{\partial t}+\boldsymbol{Q}=-\kappa \nabla T$,

where $t$ is some small positive number, which can be thought of as a relaxation time-scale for the medium (Herrera \& Santos 1997). Now plugging this back into equation (1), we have a hyperbolic equation, known as the telegrapher's equation; the problem of instantaneous propagation has been removed, but at the expense of introducing a term not clearly linked to any underlying microphysics.

The relativistic heat equation used for neutron stars and black hole space-times emerges from the same derivation as in Newtonian gravity, but with all quantities redshifted, as they are seen by a distant observer (Misner \& Sharp 1965; Thorne 1967; Van Riper 1991). The causality problem is therefore still present, but is now even more serious; it is fundamentally unacceptable in GR for any information to propagate beyond the speed of light, let alone at infinite speed. The first successful (and not ad hoc) resolution to the problem was the Israel-Stewart approach (Israel \& Stewart 1979), which posits an expansion of the entropy flux through a set of terms which encode deviations from thermal equilibrium. The various independent coefficients of this expansion need, however, to be fixed with additional constraints (either theoretical or experimental). The theory is thus rather complex, but none the less pragmatically motivated: it allows one to recover a causal heat equation, a relativistic analogue of the Cattaneo equation.

An alternative starting point is the multifluid (variational) approach of Carter - see e.g. Carter (1989), or Lopez-Monsalvo (2011) for a fuller account of the problem and relevant references. In this, entropy is regarded mathematically as a massless ${ }^{1}$ fluid, which satisfies continuity and Euler equations like any other fluid. Furthermore, the theory naturally allows for three kinds of interaction between different fluid species. One can have chemical reactions describing the creation or destruction of particles - although, in the case of entropy, the second law of thermodynamics dictates that the reaction rate must be non-negative. Secondly, dissipation is introduced through a series of scattering terms, which include scattering of the

${ }^{1}$ More precisely, a fluid composed of particles with no rest mass. usual fluid particles with the entropy 'particles' (e.g. phonons). The third, and least familiar, interaction is entrainment. This is a nondissipative coupling between two fluid species, which depends on the relative velocity between the two. In normal fluid dynamics, the momentum and velocity of a fluid are parallel; in the presence of entrainment the two can be misaligned, a phenomenon sometimes known as the Andreev-Bashkin effect (Andreev \& Bashkin 1975; Alpar, Langer \& Sauls 1984) in the context of mixed Fermi liquids.

Within the multifluid framework, it is certainly permitted to allow for entrainment between the entropy fluid and other species, but the physical reason for doing so is not initially obvious. Indeed, in the original incarnation of his multifluid model, Carter dropped these terms for simplicity. It was quickly seen, however, that the resulting theory suffered from serious instabilities (Olson \& Hiscock 1990), casting doubt on the usefulness of the framework until Priou (1991) showed that the theory was indeed stable once the entrainment terms were restored. In fact, the resulting model can be shown to be equivalent to the Israel-Stewart theory up to first order in deviations from thermal equilibrium (Lopez-Monsalvo \& Andersson 2011).

Despite its superficially obscure nature, entrainment between the entropy fluid and the matter fluid(s) in a system is also the key ingredient which allows one to derive a Cattaneo-type form of Fourier's law, and thus restore causality to heat conduction within the multifluid approach - both in Newtonian gravity and GR (LopezMonsalvo 2011). Because it acts over time-scales much shorter than those of many relativistic astrophysics problems, it might appear to be irrelevant for these. Let us sound two notes of caution, though. First, the standard heat equation will not be adequate for modelling every hot relativistic system - especially not those involving dynamic space-times or processes with short characteristic timescales. Secondly, even when it should be valid, there is still a risk that the neglect of entrainment could lead to instabilities, as for Carter's original approach. For these reasons, whilst we will indeed drop entropy entrainment during the course of deriving the heat equation, we will keep it for the first steps, to show where the explicitly causal terms lie.

\subsection{The equations of entropy dynamics}

Our starting point will be Andersson et al. (2017), hereafter AHDC, who use a general-relativistic multifluid formalism in which entropy becomes, mathematically, just another fluid. Thermal dynamics are then described by one scalar entropy equation, and one vector entropy-momentum equation. We begin, as AHDC do, in geometrized units where $G=c=1$ (so that all quantities have dimensions with powers of length alone), then restore these factors afterwards. AHDC utilize the standard ' $3+1$ split', in which four-dimensional space-time is foliated into a nested set of threedimensional spacelike hypersurfaces threaded by a set of timelike worldlines which cut through each hypersurface perpendicularly; see e.g. Thorne \& MacDonald (1982). In what follows, we will denote space-time quantities using indices $a, b$ (taking values from 0 to 3 ); and will use the indices $i, j, k$ (taking values from 1 to 3 ) to denote quantities restricted to the hypersurfaces. We will give only a minimal description of the $3+1$ split, and refer the reader to the notes of Gourgoulhon (2007) for a detailed, pedagogical description.

An observer travelling along a timelike worldline (often called an Eulerian observer) experiences proper time $\tau$ and has a 4-velocity $N$ (in index notation, $N^{a}$ ) given by $\mathrm{d} / \mathrm{d} \tau$. The relationship between an observer's proper time and the 'global time' $t$, measured by an 
observer at infinity, is encoded in the lapse $\alpha$, defined as

$\alpha \equiv \frac{\mathrm{d} \tau}{\mathrm{d} t}$

along a worldline.

The notion of time variation may be expressed in terms of the $3+1$ split, as the Lie derivative along the 4 -vector

$\boldsymbol{t}=\alpha \boldsymbol{N}+\boldsymbol{\beta}$ or $t^{a}=\alpha N^{a}+\beta^{a}$,

using a coordinate basis for the second expression. Here $\boldsymbol{\beta}$ is the 'shift vector': a 3-vector living in a spacelike hypersurface, so that

$\beta^{a} N_{a}=0$.

We may therefore denote the shift vector $\beta^{i}$ rather than $\beta^{a}$, when it does not result in mismatched indices within the same expression. The shift vector may be arbitrarily specified, aside from the restriction of being spatial. After this general summary section, we will specify to stationary and axisymmetric space-times, i.e. spacetimes with timelike and azimuthal Killing vectors. The arbitrariness of the shift vector discussed above is then very useful, because we may define it so that $\alpha \boldsymbol{N}+\boldsymbol{\beta}$ is equal to the timelike Killing vector (the natural notion of time in this context).

Now, from the lapse and shift we can split the space-time, writing $x^{a}=\left(t, x^{i}\right)$, so that the line element reads

$\mathrm{d} l^{2}=g_{a b} \mathrm{~d} x^{a} \mathrm{~d} x^{b}=-\left(\alpha^{2}-\beta_{i} \beta^{i}\right) \mathrm{d} t^{2}+2 \beta_{i} \mathrm{~d} x^{i} \mathrm{~d} t+\gamma_{i j} \mathrm{~d} x^{i} \mathrm{~d} x^{j},(7)$

where $g_{a b}$ is the space-time metric and $\gamma_{i j}$ the 3 -metric of the spatial hypersurfaces.

Returning to derivatives, we may use the linearity of the Lie derivative to show that

$\partial_{t}=\mathcal{L}_{t}=\mathcal{L}_{\alpha N+\beta}=\mathcal{L}_{\alpha N}+\mathcal{L}_{\beta}$.

Since the Lie derivative of a covariant 4-vector $v_{a}$ along some other 4-vector $u^{a}$ is given by

$\mathcal{L}_{u} v_{a}=u^{b} v_{a, b}+v_{b} u_{a}^{b}$,

we see that for a scalar $h$ :

$\mathcal{L}_{N} h=\frac{1}{\alpha}\left(\partial_{t}-\mathcal{L}_{\beta}\right) h$.

The same is also true for a covariant 3 -vector $p_{i}$. To see this, first note that since $N_{a}$ is timelike, $N_{a} q^{a}=0$ for a 3-vector $q^{i}$, and therefore $0=N_{a} q^{a}=g_{a b} N^{b} g^{a c} q_{c}=N^{b} q_{b}$. Using this result and equation (9), we then find that

$\mathcal{L}_{\alpha N} p_{i}=\alpha N^{b} p_{i, b}+p_{j}\left(\alpha N^{j}\right)_{, i}=\alpha N^{b} p_{i, b}+\alpha p_{j} N_{, i}^{j}=\alpha \mathcal{L}_{N} p_{i}$,

and so

$\mathcal{L}_{N} p_{i}=\frac{1}{\alpha}\left(\partial_{t}-\mathcal{L}_{\beta}\right) p_{i}$

In addition to the Lie derivative, we will briefly use the covariant derivative for space-time quantities, denoting it with a subscript semicolon; and will extensively use the covariant derivative projected into the hypersurfaces, $D_{i}$ (see AHDC for more details). We recall its form, in components, when acting on co- and contra-variant 3 -vectors, $p_{i}$ and $q^{i}$, respectively:

$D_{j} p_{i}=p_{i, j}-\Gamma_{j i}^{k} p_{k}$,

$D_{j} q^{i}=q_{, j}^{i}+q^{k} \Gamma_{j k}^{i}$,

where the Christoffel symbols $\Gamma_{j k}^{i}$ (associated with the projected covariant derivatives) encode the difference between covariant and partial derivatives of a 3-vector which arises from the curvature of space-time. In our context, we only have derivatives within a hypersurface, for which these symbols involve derivatives of the 3-metric $\gamma_{i j}$ only:

$\Gamma_{j k}^{i}=\frac{1}{2} \gamma^{i l}\left(\gamma_{j l, k}+\gamma_{l k, j}-\gamma_{j k, l}\right)$.

Note that the 3-metric is diagonal in the cases we consider, i.e. $\gamma^{i l}$ $=0$ for $i \neq l$.

Within the $3+1$ framework, the 4-velocity $u_{\mathrm{x}}^{a}$ of any fluid species $\mathrm{x}$ is split as follows:

$u_{\mathrm{x}}^{a}=W_{\mathrm{x}}\left(N^{a}+\hat{v}_{\mathrm{x}}^{a}\right)$,

where $\hat{v}_{\mathrm{x}}^{a}$ is the spacelike 3-velocity of the fluid within a hypersurface, as seen by an Eulerian observer, and

$W_{\mathrm{x}}=\left(1-\hat{v}_{\mathrm{x}}^{2}\right)^{-1 / 2}$

is the Lorentz factor associated with the $\mathrm{x}$-fluid. As mentioned earlier, thermal dynamics in the multifluid formalism is described quite naturally by treating entropy as a massless 'fluid' (Prix 2004). Then, the entropy flux $n_{s}^{a}$ under the $3+1$ split is given by

$n_{s}^{a}=n_{s} u_{s}^{a}=W_{s} n_{s}\left(N^{a}+\hat{v}_{s}^{a}\right)=\hat{n}_{s}\left(N^{a}+\hat{v}_{s}^{a}\right)=\hat{s}\left(N^{a}+\hat{v}_{s}^{a}\right)$,

where a hat over a symbol (e.g. $\hat{n}_{s}$ ) refers to a quantity measured by an Eulerian observer; unaccented quantities in this paper are either universal or referred to a fluid frame. Here we have recognized that the number density $\hat{n}_{s}$ of the entropy fluid - measured by an Eulerian observer - is the entropy density $\hat{s}$. Like all fluids, the entropy has a continuity equation associated with it:

$\left(n_{s}^{a}\right)_{; a}=\Gamma_{s} \geq 0$,

where $\Gamma_{s}$ is the entropy production rate. The only thing which distinguishes this continuity equation from the usual form is that the production rate must be non-negative in this case, by the second law of thermodynamics. We shall call equation (19) the entropy equation.

The other fundamental quantity is the entropy 4-momentum:

$\mu_{a}^{s}=\hat{T} N_{a}+S_{a}^{s}$,

where we have identified the time component of the 4-momentum as the temperature $\hat{T}$, and denoted the entropy 3-momentum by $S_{a}^{s}$ (which is spacelike; it satisfies $N^{a} S_{a}^{s}=0$ ). Again, in analogy with ordinary fluids, the non-conservation of the entropy 4-momentum is due to chemical reactions and frictional processes (most naturally measured by an observer in the fluid frame with some 4-velocity $u_{a}$ ), as expressed in the momentum equation:

$$
\begin{aligned}
& \left(N^{a}+v_{s}^{a}\right)\left(\mu_{a ; b}^{s}-\mu_{b ; a}^{s}\right)+\Gamma_{s} \mu_{a}^{s}=\Gamma_{s} T\left(N_{a}+v_{a}^{s}\right) \\
& \quad+\sum_{\mathrm{m} \neq s} \mathcal{R}^{s \mathrm{~m}}\left(\delta_{a}^{b}+v_{s}^{b} u_{a}\right)\left(v_{b}^{\mathrm{m}}-v_{b}^{s}\right)
\end{aligned}
$$

which may be obtained by combining equations 64,65 , and 72 from AHDC with equation (16) of this paper. Note that here, and later, we will generally refer to all fluid species other than the entropy as matter fluids (assumed to move together), with index m. Equation (21), being an intermediate algebraic step, is not the usual guise in which the momentum equation appears. None the less, we can identify the familiar features by thinking of the space-time coordinate with index 0 as time and coordinates 1,2,3 as spatial. Then, we see that equation (21) features a time-derivative of the momentum as well as its divergence - representing the notion from Newton's second law that the imbalance of forces on a fluid source its net acceleration - together with various terms which describe the 
transfer of momentum into or out of the entropy-fluid component by dissipation or reactions.

As discussed in Section 2.1, entropy entrainment is crucial for constructing a causal theory of thermal dynamics. Although we will not retain this effect later on, it is instructive to see where it features in these fundamental equations. In principle the entrainment term could then be propagated through the rest of our derivation of Fourier's law to yield an explicitly causal final heat equation. It appears in the definition of the entropy 3-momentum (see AHDC equation 96):

$S_{s}^{i}=\hat{T} \hat{v}_{s}^{i}+\sum_{\mathrm{m} \neq s} \mathcal{A}^{s \mathrm{~m}} \hat{n}_{\mathrm{m}}\left(\hat{v}_{\mathrm{m}}^{i}-\hat{v}_{s}^{i}\right)$,

from which it is easy to see that entrainment allows for the entropy momentum and velocity to be misaligned. The time-scale on which a coefficient $\mathcal{A}^{\text {sm }}$ couples the matter and entropy dynamics should therefore be some analogue of the phenomenological relaxation time-scale $\mathfrak{t}$ from the Cattaneo equation (3) (Lopez-Monsalvo 2011).

We have introduced the entropy (19) and entropy momentum (21) equations as they emerge from the multifluid framework. A major part of the work of AHDC was to rewrite and simplify these to $3+1$ forms closer to those used in numerical relativity. We refer the reader to their paper for derivations, and here simply present the required results. First, the entropy equation may be rewritten as (AHDC equation 136)

$\partial_{t}\left(\gamma^{1 / 2} \hat{s}\right)+D_{i}\left\{\alpha \gamma^{1 / 2}\left[\frac{Q^{i}}{\hat{T}}+\left(\hat{v}^{i}-\frac{\beta^{i}}{\alpha}\right) \hat{s}\right]\right\}=\alpha \gamma^{1 / 2} \Gamma_{s}$,

where $\gamma=g^{a b} \gamma_{a b}$ is the determinant of the spatial 3-metric, $\hat{v}^{i}$ the 3-velocity of a fluid-frame observer, and where we have defined a more physically motivated quantity - the heat flux - as

$$
Q^{i} \equiv \hat{s} \hat{T}\left(\hat{v}_{s}^{i}-\hat{v}^{i}\right)=\hat{s} S_{s}^{i}-\hat{s} \hat{T} \hat{v}^{i}-\hat{s} \hat{T} \sum_{\mathrm{m} \neq \mathrm{s}} \mathcal{A}^{s \mathrm{~m}} \hat{n}_{\mathrm{m}}\left(v_{\mathrm{m}}^{i}-v_{s}^{i}\right) .
$$

This quantity - analogous to the electric current - depends on the relative flow of two fluids with respect to one another. In the case of the heat flux the two fluid species are matter and entropy. Note that the vanishing of $Q^{i}$ does not imply the vanishing of $S_{s}^{i}$, nor vice versa: the heat flux vanishes when a fluid observer measures the entropy velocity as zero, whereas the entropy momentum vanishes when an Eulerian observer sees zero entropy velocity. AHDC make use of a linear-drift approximation - that the drift of the entropy fluid with respect to the matter fluid should be slow - which implies that $Q^{2} \sim\left(\hat{v}_{s}-\hat{v}\right)^{2} \ll Q$. This means that in expressions featuring different powers of $Q$, we only need to retain those terms of the lowest power. We now turn to the expression for the entropy momentum equation given in ${ }^{2}$ AHDC equation 139, which features both $Q^{i}$ and $S_{s}^{i}$ explicitly. Using equation (24) then allows us to eliminate the entropy 3-momentum in favour of the heat flux and entropy entrainment terms. If we now neglect entrainment, the result

\footnotetext{
${ }^{2}$ The above equation corrects an error from AHDC. Specifically, between their equations 78 and 80 the extrinsic curvature tensor $K_{i j}$ erroneously appears in place of the correct term, involving the trace $K$ of this tensor. The incorrect term is then propagated through their later derivations, including into their form of the entropy momentum equation 139 .
}

is

$$
\begin{aligned}
\partial_{t}\left[\gamma^{1 / 2}\left(Q_{i}+\hat{s} \hat{T} \hat{v}_{i}\right)\right]+D_{j}\left[\alpha \gamma^{1 / 2} \frac{Q^{j}}{\hat{s} \hat{T}}\left(Q_{i}+\hat{s} \hat{T} \hat{v}_{i}\right)\right] \\
\quad+D_{j}\left[\gamma^{1 / 2}\left(\alpha \hat{v}^{j}-\beta^{j}\right)\left(Q_{i}+\hat{s} \hat{T} \hat{v}_{i}\right)\right] \\
\quad+\hat{s} D_{i}\left(\alpha \gamma^{1 / 2} \hat{T}\right)-\left(\frac{Q^{j}}{\hat{T}}+\hat{s} \hat{v}^{j}\right) D_{i}\left[\alpha \gamma^{1 / 2}\left(\frac{Q_{j}}{\hat{s}}+\hat{T} \hat{v}_{j}\right)\right] \\
=\gamma^{1 / 2}\left[\alpha \mathcal{F}_{i}^{s}-\alpha K \hat{s} S_{i}^{s}+\left(Q_{j}+\hat{s} \hat{T} \hat{v}_{j}\right) D_{i} \beta^{j}\right],
\end{aligned}
$$

where the entropy entrainment terms - were they to be restored would feature together with every instance of the heat flux, except for the two contravariant $Q^{j}$ terms on the left-hand side. In equation (25), we have introduced two new quantities. The first is the trace of the extrinsic curvature

$K=\gamma^{i j} K_{i j}=\frac{1}{2 \alpha} \gamma^{i j}\left(-\gamma_{i j, t}+\beta^{k} \gamma_{i j, k}+\gamma_{k j} \beta_{, i}^{k}+\gamma_{i k} \beta_{, j}^{k}\right)$,

which is related to how a hypersurface curves within the space-time in which it is embedded; and the second is a resistive term $\mathcal{F}_{i}^{s}$ which encodes the transfer of momentum away from the entropy component due to collisions with other particle species. For clarity, let us consider only entropy-matter particle scattering, and neglect any additional resistive mechanisms (e.g. Joule heating from magneticfield decay). Under these assumptions, the collision term given by equation 142 of AHDC reduces to

$\mathcal{F}_{i}^{s}=-\frac{\mathcal{R}}{W s T}\left[Q_{i}+W^{2} \hat{v}_{i} \hat{v}^{j} Q_{j}\right]$

and the creation rate term given by AHDC equation 143 becomes

$\Gamma_{s}=\frac{\mathcal{R}}{W^{2} s^{2} T^{3}}\left[Q^{2}+W^{2}\left(\hat{v}_{j} Q^{j}\right)^{2}\right]$,

where we have defined two quantities: the Lorentz factor

$W \equiv\left(1-\hat{v}^{2}\right)^{-1 / 2}$,

and the sum of the different entropy-matter scattering coefficients

$\mathcal{R} \equiv \sum_{\mathrm{m} \neq s} \mathcal{R}^{\mathrm{m} s}$

Note that the quantities in equations (27) and (28) are related to the microphysics of the system, naturally performed in the fluid frame, and so do not have hats. The system of equations for relativistic thermal dynamics summarized above, from AHDC, is appropriate for nonlinear evolutions in dynamic space-times using a $3+1$ foliation of space-time (even though, by equation (25), the system has already ceased to be valid for studying processes on time-scales short compared with the medium's thermal relaxation). However, there are many problems for which this is unduly general. In what follows, we will explore how these equations simplify in one typical astrophysical setting.

\subsection{Rotating systems and observers}

Let us consider heat conduction in the space-time associated with a central object - either a star or a black hole - rotating uniformly at rate $\Omega$ with respect to a distant observer. Its 4 -velocity is therefore

$u^{a}=\frac{1}{\alpha}\left(\delta_{t}^{a}+\Omega \delta_{\varphi}^{a}\right)$.

We will assume that the object is isolated, so that the space-time remains stationary, and that the rotation is sufficiently slow that we may drop the second-order rotational terms which lead to deviations 
from spherical symmetry (e.g. the oblateness induced by rotation, in Newtonian or relativistic stars).

From here onwards, we will begin to restore the suppressed $G$ and $c$ factors (i.e. the dimensions) to the expressions from AHDC. By doing so, we will immediately be able to identify combinations of unfamiliar quantities from the multifluid framework with familiar transport properties, using dimensional analysis. Converting from geometrized to physical units is not especially simple, and so we establish the requisite conversions systematically, by beginning with the most fundamental quantities. To start with, it is natural to identify the spatial coordinates for our system with globally defined spherical polar coordinates, $(c t, r, \theta, \varphi)$. The line element, with geometrizing factors unsuppressed, is then

$$
\begin{aligned}
\mathrm{d} l^{2}= & -\mathrm{e}^{2 \Phi / c^{2}} c^{2} \mathrm{~d} t^{2}-2 \frac{\omega}{c} r^{2} \sin ^{2} \theta \mathrm{d} \varphi c \mathrm{~d} t+\mathrm{e}^{2 \Lambda / c^{2}} \mathrm{~d} r^{2} \\
& +r^{2} \mathrm{~d} \theta^{2}+r^{2} \sin ^{2} \theta \mathrm{d} \varphi^{2},
\end{aligned}
$$

where $\Phi=\Phi(r)$ and $\Lambda=\Lambda(r)$ are the two metric functions, and $\omega$ is the angular velocity at which frames near the central object are dragged with respect to an observer at infinity.

It is very useful to generalize the Newtonian notion of a corotating observer to the relativistic one of a zero-angular-momentum observer (ZAMO) (Bardeen 1970; Bardeen, Press \& Teukolsky 1972); these are the Eulerian observers for rotating systems. A ZAMO has a local rotational velocity of zero, and the mathematical description of physical processes is at its least complex with respect to such an observer. More specifically, one has a set of equations governing the physics of the system (e.g. a neutron star), in terms of a globally defined system of coordinates. The local coordinate system of a ZAMO is encoded in a tetrad of orthonormal basis vectors; projecting the globally defined equations on to a ZAMO's orthonormal tetrad results in a greatly simplified description of the physics. From this perspective we may regard $\omega$ as the variation, with respect to global time $t$, of the ZAMO's azimuthal coordinate:

$\omega=\frac{\mathrm{d} x^{\hat{3}}}{\mathrm{~d} x^{0}}=\frac{\mathrm{d} \hat{\varphi}}{\mathrm{d} t}$.

This quantity can be shown to be a function of the radial coordinate alone (Hartle 1967). It is given by the equation

$\frac{1}{r^{3}} \frac{\mathrm{d}}{\mathrm{d} r}\left(r^{4} \mathrm{e}^{-(\Phi+\Lambda) / c^{2}} \frac{\mathrm{d} \bar{\omega}}{\mathrm{d} r}\right)+4 \frac{\mathrm{d}}{\mathrm{d} r}\left(\mathrm{e}^{-(\Phi+\Lambda) / c^{2}}\right) \bar{\omega}=0$,

where

$\bar{\omega}=\Omega-\omega$

is the angular velocity of a fluid element as seen by a ZAMO. Now, comparing the two line elements (7) and (32) - which means identifying the ZAMO 4-velocity $u^{a}$ with the normal $N^{a}$ - we see that the lapse and shift are given by

$\alpha=\mathrm{e}^{\Phi / c^{2}} \beta^{i}=-\frac{\omega}{c} \delta_{\varphi}^{i}$.

It is now clear that the $\beta_{i} \beta^{i}=\beta^{2}$ term in the $3+1$ line element, equation (7), corresponds to second-order rotational corrections in our problem, and therefore may be neglected. We now also know that the fluid 3-velocity, as seen by a ZAMO, is

$\hat{v}^{i}=\mathrm{e}^{-\Phi / c^{2}} \bar{\omega} \delta_{\varphi}^{i}$ and so all Lorentz factors reduce to unity, since

$$
\begin{aligned}
\left(1-\frac{\hat{v}^{2}}{c^{2}}\right)^{-1 / 2} & =\left(1-\mathrm{e}^{-2 \Phi / c^{2}} \frac{\bar{\omega}^{2}}{c^{2}}\right)^{-1 / 2} \\
& =1+\frac{1}{2 !} \mathrm{e}^{-2 \Phi / c^{2}} \frac{\bar{\omega}^{2}}{c^{2}}+\cdots \approx 1,
\end{aligned}
$$

using our assumption of slow rotation.

A brief calculation using equation (26) shows that the extrinsic curvature $K_{i j}$ vanishes for a non-rotating star, but in a rotating system has one independent non-zero component, which in physical units is (using primes to denote derivatives with respect to $r$ )

$K_{r \varphi}=-\frac{1}{2} \mathrm{e}^{-\Phi / c^{2}} r^{2} \sin ^{2} \theta \omega^{\prime}(r)=K_{\varphi r}$

since $K_{i j}$ is symmetric (Gourgoulhon 2007). To get the trace $K$ of this quantity, however, we must contract it with the 3-metric $\gamma^{i j}$, which is diagonal - and therefore $K=0$.

\subsection{Physical quantities and their dimensions}

Let us pause to discuss the quantities involved in the equations for thermal dynamics and also their dimensions, which will help in the interpretation of physical quantities later on in our derivations. We denote by $M, L, T$, and $\Theta$ the dimensions of mass, length, time, and temperature. The physical dimensions of the two basic thermal quantities, $T$ and $s$, are

$$
\begin{aligned}
{\left[T_{\text {phys }}\right] } & =\Theta\left[s_{\text {phys }}\right]=[\mathcal{S}] \mathrm{L}^{-3}=\mathrm{ML}^{2} \mathrm{~T}^{-2} \Theta^{-1} \times \mathrm{L}^{-3} \\
& =\mathrm{ML}^{-1} \mathrm{~T}^{-2} \Theta^{-1},
\end{aligned}
$$

where $\mathcal{S}$ is the true entropy (i.e. not per unit volume). In standard geometrized units for temperature-independent problems, one sets $G=c=1$, and all quantities have dimensions which are powers of $\mathrm{L}$. It is possible to extend this to relativistic thermal dynamics by setting $G=c=k_{\mathrm{B}}=1$, where $k_{\mathrm{B}}$ is the Boltzmann constant; e.g. one divides entropy by $k_{\mathrm{B}}$, so that its geometrized form is dimensionless. Since no algebra from AHDC involved factors of $k_{\mathrm{B}}$ anyway though, their equations are the same in either system of geometrized units. Accordingly, we will proceed with the simpler $G=c=1$ system, allowing the dimensions of each quantity to contain powers of both $\mathrm{L}$ and $\Theta$.

In a $G=c=1$ unit system, $T$ remains the same, but we must multiply $s$ by a prefactor combination of $G$ and $c$ to eliminate T and M:

$s_{\text {geom }}=\frac{G}{c^{4}} s_{\text {phys }} \Rightarrow\left[s_{\text {geom }}\right]=\mathrm{L}^{-2} \Theta^{-1}$.

Next, all velocities in geometrized units are of the form

$v_{\mathrm{geom}}^{i}=\frac{v_{\mathrm{phys}}^{i}}{c}$

and so are dimensionless. The heat flux $Q^{i}$ in geometrized units is therefore

$Q_{\mathrm{geom}}^{i}=s_{\mathrm{geom}} T\left(v_{s}^{i}-v^{i}\right)_{\mathrm{geom}}=\frac{G}{c^{5}} s_{\mathrm{phys}} T\left(v_{s}^{i}-v^{i}\right)_{\mathrm{phys}}$

and has dimensions

$\left[Q_{\text {geom }}^{i}\right]=\mathrm{L}^{-2}$.

If we divide through by the geometrizing prefactor we get

$\left[Q_{\mathrm{phys}}^{i}\right]=[c]^{5}[G]^{-1}\left[Q_{\mathrm{geom}}^{i}\right]=\mathrm{MT}^{-3}$,

which are indeed the expected physical units for heat flux. Next, because our physical coordinates are $(c t, r, \theta, \varphi)$, time derivatives 
must contain a $1 / c$ factor, which is suppressed in geometrized units:

$\left(\partial_{t}\right)^{\text {geom }}=\frac{1}{c}\left(\partial_{t}\right)^{\text {phys }}$.

This means a time derivative in geometrized units has dimensions $\mathrm{L}^{-1}$. From (23) we then see that

$\left[\Gamma_{s}^{\text {geom }}\right]=\left[\left(\partial_{t} s\right)^{\text {geom }}\right]$

and so

$\Gamma_{s}^{\text {geom }}=\frac{G}{c^{5}} \Gamma_{s}^{\text {phys }}$.

Using equation (25) we can determine that in geometrized units

$\left[\mathcal{F}_{i}^{s}\right]=\left[s D_{i} \alpha T\right]=\mathrm{L}^{-2} \Theta^{-1} \times \mathrm{L}^{-1} \times \Theta=\mathrm{L}^{-3}$,

and so

$\left(\mathcal{F}_{i}^{s}\right)^{\text {geom }}=\frac{G}{c^{4}}\left(\mathcal{F}_{i}^{s}\right)^{\text {phys }}$.

Finally, by equation (27) we have

$[\mathcal{R}]=\left[\mathcal{F}_{i}^{s}\right][s][T]\left[Q_{i}\right]^{-1}=\mathrm{L}^{-3} \mathrm{~L}^{-2} \Theta^{-1} \Theta\left(\mathrm{L}^{-2}\right)^{-1}=\mathrm{L}^{-3}$.

Again restoring the suppressed geometric prefactors, we have

$\mathcal{R}_{\text {geom }}=\frac{G}{c^{3}} \mathcal{R}_{\text {phys }}$

i.e.

$\left[\mathcal{R}_{\text {phys }}\right]=\mathrm{ML}^{-3} \mathrm{~T}^{-1}$.

\section{FOURIER'S LAW}

The standard form of Fourier's law, equation (2), relates the heat flux to the temperature gradient, with the thermal conductivity as the constant of proportionality. If heat conduction is different in different directions - e.g. due to the effect of a magnetic field one needs to replace the scalar conductivity with a tensorial one $\kappa$, so that $\boldsymbol{Q}=-\boldsymbol{\kappa} \cdot \nabla T$ (Urpin \& Yakovlev 1980). As discussed in Section 2.1, relativistic forms of Fourier's law and the heat equation are by no means novel (e.g. Van Riper 1991; Aguilera et al. 2008), and date back to at least the 1960s (Misner \& Sharp 1965; Thorne 1967). However, these have been generalized from their Newtonian counterparts in a simple way, essentially by replacing flat-space quantities in the derivations of these equations by redshifted ones (i.e. the locally measured value of some quantity in a spherical space-time needs to be multiplied by a factor of $\mathrm{e}^{\Phi / c^{2}}$ to yield the value seen by a distant observer). This makes it difficult to see how causality could be restored to the heat equation, or what new terms would appear in a more complex relativistic system. Here, by contrast, we aim to derive relativistic forms of Fourier's law and the heat equation from the manifestly causal multifluid formalism, in which the route to generalizing the model is also clear. We will first recover the expected equation for Fourier's law in a non-rotating star, then will consider the problem with terms at first order in the rotation.

First, let us recall from AHDC the results:

$$
\begin{aligned}
{\left[(-g)^{1 / 2}\right]_{; a}=\left(\alpha \gamma^{1 / 2}\right)_{; a}=0 \quad D_{i}\left(\alpha \gamma^{1 / 2}\right) } & =\partial_{i}\left(\alpha \gamma^{1 / 2}\right)-\Gamma_{j i}^{j} \gamma^{1 / 2} \\
& =0 .
\end{aligned}
$$

In addition

$\left(\gamma^{1 / 2}\right)_{, t}=\left(\alpha \gamma^{1 / 2}\right)_{, t}=0$

by the stationarity of the space-time we consider; recall that we are not allowing for the space-time itself to evolve here. Given these, we may pull these quantities out of the covariant and time derivatives, and cancel them. The entropy momentum equation (25) in geometrized form then reduces to

$$
\begin{aligned}
& \frac{1}{\alpha} \partial_{t}\left(Q_{i}+\hat{s} \hat{T} \hat{v}_{i}\right)+\left(\hat{v}^{j}-\frac{\beta^{j}}{\alpha}\right) D_{j}\left(Q_{i}+\hat{s} \hat{T} \hat{v}_{i}\right) \\
& \quad+D_{j}\left[\frac{Q^{j}}{\hat{s} \hat{T}}\left(Q_{i}+\hat{s} \hat{T} \hat{v}_{i}\right)\right]+\hat{s} D_{i} \hat{T}-\left(\frac{Q^{j}}{\hat{T}}+\hat{s} \hat{v}^{j}\right) \\
& \quad \times D_{i}\left(\frac{Q_{j}}{\hat{s}}+\hat{T} \hat{v}_{j}\right)=\mathcal{F}_{i}^{s}+\frac{1}{\alpha}\left(Q_{j}+\hat{s} \hat{T} \hat{v}_{j}\right) D_{i} \beta^{j},
\end{aligned}
$$

where we have used the fact that both our shift vector and the (rigidly rotating) fluid flow are divergence-free, $D_{j} \beta^{j}=D_{j} \hat{v}^{j}=0$, and that $K=0$. Comparing equations (12) and (13), we see that the first two terms of the above equation (56) may be rewritten as follows:

$$
\begin{aligned}
\frac{1}{\alpha}\left(\partial_{t}\right. & \left.-\beta^{j} D_{j}\right)\left(Q_{i}+\hat{s} \hat{T} \hat{v}_{i}\right)+\hat{v}^{j} D_{j}\left(Q_{i}+\hat{s} \hat{T} \hat{v}_{i}\right) \\
= & \mathcal{L}_{N}\left(Q_{i}+\hat{s} \hat{T} \hat{v}_{i}\right)+\frac{1}{\alpha}\left(Q_{j}+\hat{s} \hat{T} \hat{v}_{j}\right) \beta_{, i}^{j} \\
& +\frac{1}{\alpha} \beta^{j} \Gamma_{j i}^{k}\left(Q_{k}+\hat{s} \hat{T} \hat{v}_{k}\right)+\hat{v}^{j} D_{j}\left(Q_{i}+\hat{s} \hat{T} \hat{v}_{i}\right) .
\end{aligned}
$$

Next, we expand and rearrange the covariant derivative from the right-hand side of equation (56):

$\frac{1}{\alpha}\left(Q_{j}+\hat{s} \hat{T} \hat{v}_{j}\right) D_{i} \beta^{j}=\frac{1}{\alpha}\left(Q_{j}+\hat{s} \hat{T} \hat{v}_{j}\right) \beta_{, i}^{j}+\frac{1}{\alpha}\left(Q_{k}+\hat{s} \hat{T} \hat{v}_{k}\right) \beta^{j} \Gamma_{j i}^{k}$,

where we have relabelled the indices and used the fact that the Christoffel symbols are symmetric in the lower two indices. Inserting these last two results into the entropy momentum equation (still in geometrized form), a number of terms cancel and we are left with

$$
\begin{aligned}
& \frac{1}{\hat{s}} \mathcal{L}_{N}\left(Q_{i}+\hat{s} \hat{T} \hat{v}_{i}\right)+\frac{1}{\hat{s}} \hat{v}^{j} D_{j} Q_{i}+\frac{1}{\hat{s}} D_{j}\left(Q^{j} \hat{v}_{i}\right)-\frac{Q^{j}}{\hat{s} \hat{T}} D_{i}\left(\hat{v}_{j} \hat{T}\right) \\
& \quad+\hat{v}^{j} D_{i}\left(\frac{Q_{j}}{\hat{s}}\right)+D_{i} \hat{T}=\frac{1}{\hat{s}} \mathcal{F}_{i}^{s},
\end{aligned}
$$

where we have also made use of the result $Q^{2} \ll Q$ (which follows from the linear-drift approximation; see AHDC) in order to drop terms quadratic in the heat flux. The Lie derivative of $\hat{s} \hat{T} \hat{v}_{i}$ may be rewritten using a couple of results derived later in this paper. Rather than breaking the flow here to discuss these here, we refer the reader forward to Section 4. Specifically, we use equations (71) and (85) together with the assumption of rigid, slow rotation, to find that

$\mathcal{L}_{N}\left(\hat{s} \hat{T} \hat{v}_{i}\right)=\left(1+\frac{\hat{s}}{C_{V}}\right) \hat{T} \hat{v}^{i}\left[\Gamma_{s}-D_{j}\left(\frac{Q^{j}}{\hat{T}}\right)\right]$.

We see that this term, like many others in equation (59), is proportional to $\hat{v} Q$. But since $\hat{v} \sim \hat{v}_{s}$ (linear-drift approximation), we know that

$\hat{v} Q \propto \hat{v}\left(\hat{v}-\hat{v}_{s}\right) \propto \hat{v}^{2}$

and so for consistency with our slow-rotation approximation all of these terms should be neglected. The entropy momentum equation in physical units then reduces to

$\frac{1}{c^{2} \hat{s}} \mathcal{L}_{N} Q_{i}+D_{i} \hat{T}=-\frac{\mathcal{R}}{s^{2} T} Q_{i}$,

where we have restored the suppressed $G$ and $c$ factors and plugged in the explicit form of the scattering term (27). 


\subsection{Non-rotating limit}

We can understand equation (62) by looking at the limit in which $\omega \rightarrow 0$. This corresponds to no frame dragging, and therefore no rotation in relativistic gravity. We find that

$\frac{1}{c^{2} \alpha \hat{s}} \partial_{t} Q_{i}+D_{i} \hat{T}=-\frac{\mathcal{R}}{s^{2} T} Q_{i}$.

Comparing with the usual form of Fourier's law (2), let us identify the following quantity as the heat conductivity $\kappa$ :

$\kappa \equiv \frac{s^{2} T}{\mathcal{R}}$.

We can use dimensional analysis as a consistency check of this definition, using results from Section 2.4; we find that $[\kappa]=$ $\mathrm{MLT}^{-3} \Theta^{-1}$, which are indeed the expected physical units. Now, equation (63) becomes

$\frac{1}{c^{2} \alpha \hat{s}} \partial_{t} Q_{i}+D_{i} \hat{T}=-\frac{1}{\kappa} Q_{i}$

This is not quite what we want though: the usual form of Fourier's law has no time dependence, whereas here we find a time derivative of the heat flux. Equally though, this factor does not render equation (65) the kind of stable, causal expression resulting from the inclusion of the entropy entrainment - despite its superficial resemblance to equation (3) (see e.g. Lopez-Monsalvo 2011). This is because the prefactor of $\partial_{t} Q_{i}$ in equation (65) is not 'tuneable' and has no connection with the medium through which heat propagates. Only in the limit of Newtonian dynamics, where $Q / c \propto v_{s} / c \rightarrow 0$, does the time-derivative term vanish automatically.

At this point we see that in GR we can recover the standard Fourier's law only if the heat flow is approximately steady on a thermal time-scale. More precisely, we want the time-scale $\tau_{Q}$ for variations in the heat flux to satisfy:

$\tau_{Q} \gg \frac{\kappa}{c^{2} \alpha \hat{s}}$,

but the $c^{2}$ factor means that this assumption ought to be quite safe - at least for processes on secular time-scales. Finally then, we can reach the desired result:

$D_{i} \hat{T}=-\frac{1}{\kappa} Q_{i}$.

\subsection{Slow rotation}

Having thus identified the heat conductivity from the Newtonian limit of the entropy momentum equation, let us return to the general case, equation (62). For the same reasons as in the non-rotating case, we again want to assume the heat flow is steady over dynamical time-scales. The natural notion of time variation in the foliation framework, however, is not with respect to global time, $\partial_{t}$, but rather a local expression given by the Lie derivative $\mathcal{L}_{N}$ along the normal to the hypersurfaces:

$\mathcal{L}_{N}=\frac{1}{\alpha}\left(\partial_{t}-\mathcal{L}_{\beta}\right)$

We therefore neglect this term from the left-hand side of equation (62), substituting in equation (64) for $\kappa$, to find that

$D_{i} \hat{T}=-\frac{1}{\kappa} Q_{i}$,

as in the non-rotating case. Thus, Fourier's law will only differ from its form in a non-rotating star for the case of rapid rotation.

\section{THE HEAT EQUATION}

We used the entropy momentum equation (25) to derive Fourier's law, above. Now we use the corresponding scalar entropy equation (23) to derive the heat equation. As for the derivation of Fourier's law, we begin by taking the $\gamma^{1 / 2}$ and $\alpha \gamma^{1 / 2}$ factors out of the covariant and time derivatives and cancelling the former, leaving us with

$\partial_{t} \hat{s}+\alpha D_{i}\left(\frac{Q^{i}}{\hat{T}}\right)-\left(\alpha \hat{v}^{i}-\beta^{i}\right) D_{i} \hat{s}=\alpha \Gamma_{s}$,

where we have again used the fact that $D_{j} \beta^{j}=D_{j} \hat{v}^{j}=0$. Within the $3+1$ approach, it is natural to re-express equation (70) in terms of the Lie derivative along the timelike normal $N$ :

$\mathcal{L}_{N} \hat{s}-\hat{v}^{i} D_{i} \hat{s}+D_{i}\left(\frac{Q^{i}}{\hat{T}}\right)=\Gamma_{s}$,

where the shift vector is given by the ZAMO rotational frequency, and this is the form of the entropy equation we will use. Note, however, that the equation takes an even simpler form when expressed in terms of a 'material derivative' $\mathcal{L}_{\boldsymbol{u}} \equiv \alpha^{-1}\left(\partial_{t}-\Omega \delta_{\varphi}^{i} D_{i}\right)$ with respect to a corotating fluid observer (rather than a ZAMO):

$\mathcal{L}_{u} \hat{s}+D_{i}\left(\frac{Q^{i}}{\hat{T}}\right)=\Gamma_{s}$.

Note that these various forms of the entropy equation are all the same in both physical and geometrized units; in the geometrized case each of the terms has a factor of $G / c^{5}$ suppressed, and so these may be cancelled. Now, by virtue of our slow-rotation approximation (meaning that $\hat{v}^{2}$ terms are negligible and $W=1$ ), the entropy creation rate from equation (28) may be simplified to

$\Gamma_{s}=\frac{\mathcal{R}}{s^{2} T^{3}} Q^{2}=\frac{Q^{2}}{\kappa T^{2}}$,

which, together with the second law of thermodynamics, implies that $\mathcal{R}>0$. Although the entropy creation rate is proportional to $Q^{2}$ and therefore small compared with $Q$, it cannot be neglected, since it is not manifestly smaller than other terms in the entropy equation. The next part of the strategy will be to rewrite terms involving derivatives of the entropy, using the first law of thermodynamics:

$\mathrm{d} \mathcal{U}=T \mathrm{~d} \mathcal{S}-P \mathrm{~d} V+\sum_{\mathrm{x} \neq s} \mu_{\mathrm{x}} \mathrm{d} \mathcal{N}_{\mathrm{x}}$

where $\mathcal{S}$ and $\mathcal{U}$ are the true entropy and internal energy (as opposed to the quantities per unit volume we use elsewhere), $P$ is pressure, $V$ volume, and $\mathcal{N}_{\mathrm{x}}$ the number of x-particles. Let us assume the total number $\mathcal{N}$ of matter particles is conserved, so that $\mathrm{d} \mathcal{N}=0$; then, for a system with a single species of matter particle, the third term becomes $\mu \mathrm{d} \mathcal{N}=0$. In the case of multiple particle species, we get a similar result if we make the additional assumption of chemical equilibrium; then $d \mathcal{N}_{x}=0$ for each species, and so the first law becomes

$\mathrm{d} \mathcal{U}=T \mathrm{~d} \mathcal{S}-P \mathrm{~d} V$.

From equations (71) and (75) we will derive the heat equation first for a non-rotating star, to show that we recover the expected result, and then for the case of slow rotation. 


\subsection{Non-rotating limit}

Taking the time derivative of the first law (75) per unit volume, we find that

$\left.\frac{\partial U}{\partial t}\right|_{V}=\left.T \frac{\partial s}{\partial t}\right|_{V}$,

where $U$ is internal energy per unit volume, and we recall that $s$ is entropy per unit volume. Now expand the left-hand side of this expression using the chain rule:

$\left.\frac{\partial U}{\partial t}\right|_{V}=\left.\left.\left.\frac{\partial U}{\partial T}\right|_{V} \frac{\partial T}{\partial t}\right|_{V} \equiv C_{V} \frac{\partial T}{\partial t}\right|_{V}$,

using the definition of $C_{V}$. Note that we do not have an additional $\partial U / \partial s$ term from applying the chain rule in equation (77), because $s$ and $T$ are thermodynamic conjugate pairs, and we may regard $U$ as being a function of either variable. Now comparing the above two equations, we can eliminate $\partial_{t} \hat{s}$ in favour of $\partial_{t} \hat{T}$ in the entropy equation (70), which for constant-volume processes becomes

$C_{V} \partial_{t} \hat{T}+\alpha \hat{T} D_{i}\left(\frac{Q^{i}}{\hat{T}}\right)=\frac{Q^{2}}{\kappa T^{2}}$

Use of the product rule on this expression then gives

$C_{V} \partial_{t} \hat{T}+\alpha D_{i} Q^{i}-\alpha \frac{Q^{i}}{\hat{T}} D_{i} \hat{T}=\frac{Q^{2}}{\kappa T^{2}}$.

Rewriting the third term with Fourier's law (67), we have

$\alpha \frac{Q^{i}}{\hat{T}} D_{i} \hat{T}=\alpha \frac{Q^{2}}{\kappa T}$

since $\hat{T}=W T \approx T$, which cancels with the right-hand-side term of (79). Then, by additionally using $D_{i}\left(\alpha \gamma^{1 / 2}\right)=D_{i}\left(\gamma^{1 / 2}\right)=0$ on the resulting expression, we can manipulate it as follows:

$$
\begin{aligned}
C_{V} \alpha \gamma \partial_{t} \hat{T} & =\alpha^{2} \gamma D_{i}\left(\kappa D^{i} \hat{T}\right)=D_{i}\left[\alpha \gamma^{1 / 2} \kappa D^{i}\left(\alpha \gamma^{1 / 2} \hat{T}\right)\right] \\
& =\gamma D_{i}\left[\alpha \kappa D^{i}(\alpha \hat{T})\right],
\end{aligned}
$$

so giving us

$C_{V} \partial_{t}\left(\mathrm{e}^{\Phi} \hat{T}\right)=D_{i}\left[\mathrm{e}^{\Phi} \kappa D^{i}\left(e^{\Phi} \hat{T}\right)\right]$ or $C_{V} \partial_{t}\left(\mathrm{e}^{\Phi} \hat{T}\right)=\nabla \cdot\left[\mathrm{e}^{\Phi} \kappa \nabla\left(\mathrm{e}^{\Phi} \hat{T}\right)\right]$,

which is the usual way in which the relativistic heat equation is presented, in terms of the redshifted temperature $\mathrm{e}^{\Phi} \hat{T}$.

\subsection{Slow rotation}

For rotating stars the logic used in deriving the heat equation is the same, but because the space-time is no longer static we again need to generalize the notion of time derivative to be the Lie derivative along the normal vector [see equation (68)]. With this concept, equation (76) from the static case generalizes to

$\left.\mathcal{L}_{N} U\right|_{V}=\left.T \mathcal{L}_{N} S\right|_{V}$

and the chain rule gives

$\left.\mathcal{L}_{N} U\right|_{V}=\left.\left.\frac{\partial U}{\partial T}\right|_{V} \mathcal{L}_{N} T\right|_{V}=\left.C_{V} \mathcal{L}_{N} T\right|_{V}$.

Combining these last two equations, as for the non-rotating case, gives

$\mathcal{L}_{N} s=\frac{C_{V}}{T} \mathcal{L}_{N} T$
We now return to the entropy equation (71), expanding the covariant derivatives with the product rule to show that

$\mathcal{L}_{N} \hat{s}-\hat{v}^{i} D_{i} \hat{s}+D_{i}\left(\frac{Q^{i}}{\hat{T}}\right)=\frac{\mathcal{R}}{s^{2} T^{2}} Q^{2}=\frac{1}{\kappa T} Q^{2}$.

Now plugging Fourier's law [equation (69)] and the definition of $\mathcal{L}_{N}$ into equation (86), we find that

$\hat{T}\left(\mathcal{L}_{N}-\hat{v}^{i} D_{i}\right) \hat{s}+D_{i} Q^{i}=\frac{Q^{2}}{\kappa T}+\frac{1}{\hat{T}} Q^{i} D_{i} \hat{T}=0$,

again exploiting $T \approx \hat{T}$. The second half of the first term above corresponds to the entropy being advected with the rotating fluid flow, and simplifies to

$\hat{v}^{i} D_{i} \hat{s}=\frac{1}{\alpha} \frac{\partial \hat{s}}{\partial \varphi}=\frac{C_{V}}{\alpha \hat{T}} \frac{\partial \hat{T}}{\partial \varphi}$,

by the same arguments which gave equations (76) and (77). Using this result and equation (85) to replace $\hat{s}$ with $\hat{T}$ in equation (87) then yields the heat equation for a rotating star,

$C_{V}\left(\mathcal{L}_{N}-\frac{\Omega}{\alpha} \frac{\partial}{\partial \varphi}\right) \hat{T}=-D_{i} Q^{i}$,

or, by expanding $\mathcal{L}_{N}$ and using Fourier's law,

$$
\begin{aligned}
C_{V}\left(\frac{\partial \hat{T}}{\partial t}-\bar{\omega} \frac{\partial \hat{T}}{\partial \varphi}\right) & =D_{i}\left(\kappa D^{i} \hat{T}\right) \text { or } C_{V}\left(\frac{\partial \hat{T}}{\partial t}-\bar{\omega} \frac{\partial \hat{T}}{\partial \varphi}\right) \\
& =\nabla \cdot(\kappa \nabla \hat{T}) .
\end{aligned}
$$

Note that in the limit of zero diffusion $(\kappa=0)$, the equation above describes a temperature distribution depending on the quantity $(\varphi+$ $\bar{\omega} t)$, as expected: the temperature moves forwards with angular velocity $\bar{\omega}=\Omega-\omega$ with respect to a ZAMO.

We have shown that one recovers the expected heat equation if only the lowest order rotational corrections are kept. Next, we briefly make contact with astrophysics, by considering one particular physical situation with direct relevance for neutron-star observations.

\section{ROTATIONAL MODULATION OF A NEUTRON-STAR HOTSPOT}

There are a number of observations of hotspots on neutron stars which show modulation in time. In some cases the frequency at which they are modulated is the only way to determine their rotation frequency (Strohmayer, Zhang \& Swank 1997). Some neutron stars accreting in low-mass X-ray binary systems produce X-ray bursts due to thermonuclear burning in the neutron-star ocean, and in some cases the bursts display almost coherent oscillations - typically in the range $\sim 300-600 \mathrm{~Hz}$ (Watts 2012). These oscillations are believed to be related either to modes of the neutron-star ocean (Heyl 2004), or to an essentially confined hotspot (Cumming \& Bildsten 2000) - though there are challenges with either model in explaining the small frequency drifts of $\sim 1 \mathrm{~Hz}$ seen in the burst oscillations. In either case, the characteristic frequency is believed to be due to rotational modulation, which implicitly assumes that an observer at infinity really sees a hotspot on the stellar surface moving at the rotation rate. Let us close by considering how reasonable this is for rapidly rotating neutron stars.

We have seen that for slow rotation - retaining only terms linear in the rotational frequency - the heat equation takes the form of equation (90). The frame-dragging term on the left-hand side of this equation tells us that a hotspot fixed on the surface of a neutron star will be rotationally modulated for a ZAMO at a frequency of 
$\Omega-\omega$. However, the ZAMO itself moves at $+\omega$ with respect to an observer at infinity - so, for this latter observer the hotspot is indeed rotationally modulated at the expected frequency of $\Omega$. We have not studied more rapidly rotating stars here, for which terms at order $\Omega^{2}$ should be retained, but there is no reason for the modulation of a hotspot to be affected by the oblate shape induced by order- $\Omega^{2}$ terms. The other class of higher order terms is those proportional to $Q \Omega$, neglected by us just before equation (62). Since they describe a coupling of the stellar rotation with the heat flux, it is indeed possible that these terms would alter a hotspot's motion on a rapidly rotating star.

In conclusion, within the slow-rotation approximation a hotspot is indeed seen modulated at exactly the stellar rotation rate. Even the most rapidly rotating neutron stars known are still rather 'slow', in the sense that their rotational kinetic energy is small compared with the gravitational binding energy, so our heat equation is probably adequate for describing all known neutron stars (with the caveat that we had to make various physical assumptions to derive it). There remains the possibility, however, that higher order couplings will produce a hotspot rotating at a slightly different rate from the star.

\section{SUMMARY}

We have shown how the standard form of the relativistic heat equation follows from a causal theory of heat propagation, in which the entropy is treated as a fluid. At first order in rotation, the only correction is the expected one that the temperature is advected with the fluid flow. We have not studied the second-order problem, but speculate that it may result in coupling between the heat flow and the rotation.

One benefit in having performed our detailed derivation is that it demonstrates how the heat equation relies implicitly on various assumptions which will not be safe in all astrophysical situations. The dynamical time-scale for the problem at hand must be long compared to the thermal relaxation time of the medium, or equivalently the time-scale on which entrainment couples the entropy and matter fluids. Equally, the heat flux must be approximately steady over short time-scales. Even in situations where these conditions are met, we should be mindful that a thermal evolution relying on the standard heat equation could still suffer genuine instabilities connected with the neglect of entropy entrainment.

It is not so surprising that the usual form of the heat equation may be recovered in the limiting case described above, where thermal information propagates almost 'instantaneously' - compared with any fluid dynamics - and where the space-time is stationary. For more dynamical situations, however, the equations presented here are not applicable - and a future goal for the numerical simulations of hot relativistic systems should be to evolve the entropy dynamics directly.

\section{ACKNOWLEDGEMENTS}

SKL acknowledges support from the European Union's Horizon 2020 research and innovation programme under the Marie
Skłodowska-Curie grant agreement No. 665778, via fellowship UMO-2016/21/P/ST9/03689 of the National Science Centre, Poland. Both authors acknowledge support from STFC via grant number ST/M000931/1, and from NewCompStar (a COST-funded Research Networking Programme). We thank the referee for an insightful report, which helped considerably in improving this paper.

\section{REFERENCES}

Aguilera D. N., Pons J. A., Miralles J. A., 2008, A\&A, 486, 255

Alpar M. A., Langer S. A., Sauls J. A., 1984, ApJ, 282, 533

Andersson N., Hawke I., Dionysopoulou K., Comer G. L., 2017, Class. Quantum Gravity, 34, 125003

Andreev A. F., Bashkin E. P., 1975, Sov. J. Exp. Theor. Phys., 42, 164

Bardeen J. M., 1970, ApJ, 162, 71

Bardeen J. M., Press W. H., Teukolsky S. A., 1972, ApJ, 178, 347

Burrows A., Lattimer J. M., 1986, ApJ, 307, 178

Carter B., 1989, in Anile A. M., Choquet-Bruhat Y., ed., in Relativistic Fluid Dynamics. Springer-Verlag, Berlin

Cumming A., Bildsten L., 2000, ApJ, 544, 453

Cumming A., Brown E. F., Fattoyev F. J., Horowitz C. J., Page D., Reddy S., 2017, Phys. Rev. C, 95, 025806

Gourgoulhon E., 2007, preprint (arXiv:gr-qc/0703035)

Govender M., Maharaj S. D., Maartens R., 1998, Class. Quantum Gravity, 15,323

Hartle J. B., 1967, ApJ, 150, 1005

Herrera L., Santos N. O., 1997, MNRAS, 287, 161

Heyl J. S., 2004, ApJ, 600, 939

Israel W., Stewart J. M., 1979, Ann. Phys., 118, 341

Lopez-Monsalvo C. S., 2011, Covariant Thermodynamics and Relativity, $\mathrm{PhD}$ thesis, Univ. Southampton

Lopez-Monsalvo C. S., Andersson N., 2011, Proc. R. Soc. A, 467, 738

Miralles J. A., Van Riper K. A., Lattimer J. M., 1993, ApJ, 407, 687

Misner C. W., Sharp D. H., 1964, Phys. Rev., 136, 571

Misner C. W., Sharp D. H., 1965, Phys. Lett., 15, 279

Negreiros R., Schramm S., Weber F., 2017, A\&A, 603, A44

Olson T. S., Hiscock W. A., 1990, Phys. Rev. D, 41, 3687

Priou D., 1991, Phys. Rev. D, 43, 1223

Prix R., 2004, Phys. Rev. D, 69, 043001

Ressler S. M., Tchekhovskoy A., Quataert E., Chandra M., Gammie C. F., 2015, MNRAS, 454, 1848

Sekiguchi Y., 2010, Prog. Theor. Phys., 124, 331

Shibata M., Taniguchi K., Uryu K., 2005, Phys. Rev. D, 71, 084021

Strohmayer T. E., Zhang W., Swank J. H., 1997, ApJ, 487, L77

Thorne K. S., 1967, in DeWitt C., Schatzmann E., Véron P., eds, in HighEnergy Astrophysics. Gordon \& Breach, New York

Thorne K. S., MacDonald D., 1982, MNRAS, 198, 339

Urpin V. A., Yakovlev D. G., 1980, Sov. Astron. 24, 425

Van Riper K. A., 1991, ApJS, 75, 449

Watts A., 2012, Ann. Rev. Astron. Astrophys., 50, 609

Woosley S. E., Heger A., Weaver T. A., 2002, Rev. Mod. Phys., 74, 1015

Yuan F., Narayan R., 2014, Ann. Rev. Astron. Astrophys., 52, 529

This paper has been typeset from a $\mathrm{T}_{\mathrm{E}} \mathrm{X} / \mathrm{LT}_{\mathrm{E}} \mathrm{X}$ file prepared by the author. 